\title{
B7-H3 Promotes Proliferation and Migration of Colorectal Cancer Cells by Regulating PYCR1 in the Proline Metabolism
}

\author{
J in $Y^{1,2}$, Jiang $X^{2}$, Wu $R^{2}$, Liu $H^{2}$, Zhang $T^{1}, X^{1} X^{1}$, \\ Zhang $L^{3}$, Wang $X^{1,2}$, Hua $D^{1,2 \#}$ and Yong Mao ${ }^{1,2 *}$ \\ ${ }^{1}$ Department of Oncology, Affiliated Hospital of Jiangnan \\ University, Wuxi, Jiangsu, China \\ ${ }^{2}$ Wuxi Medical College, Jiangnan University, Wuxi, \\ Jiangsu, China \\ ${ }^{3}$ Zhongshan Hospital Affiliated to Xiamen University, \\ Xiamen University, Xiamen, Fujian, China \\ "Contributed Equally to this Work \\ *Corresponding author: Yong Mao, Department of \\ Oncology, Affiliated Hospital of Jiangnan University, \\ Wuxi, Jiangsu, China
}

Received: August 09, 2021; Accepted: September 06, 2021; Published: September 13, 2021

\begin{abstract}
Proline metabolism plays an essential role in tumor development; however, its underlying mechanism in $\mathrm{CRC}$ remains elusive. $\mathrm{B} 7-\mathrm{H} 3$, an immune checkpoint member of the B7 immunoregulatory family, is aberrantly overexpressed in a wide variety of malignancies and is associated with a poor prognosis. In this study, we found that overexpression of B7-H3 effectively enhanced proline consumption rate and reduced glutamate production, while knockout of $\mathrm{B} 7-\mathrm{H} 3$ had inverse effects. Moreover, we also identified that $\mathrm{B} 7-\mathrm{H} 3$ increased proline consumption and decreased glutamate production by promoting the expression of Pyrroline5-carboxylate reductase 1 (PYCR1) in CRC cells and that PYCR1 is a crucial mediator of $\mathrm{B} 7-\mathrm{H} 3$-induced $\mathrm{CRC}$ proliferation and migration. Overexpression of PYCR1 or treatment of cells with PYCR1 inhibitors could reverse B7-H3-induced proline metabolism and $\mathrm{B} 7-\mathrm{H} 3$-induced tumor cell proliferation and migration. Furthermore, we confirmed the positive correlation between B7-H3 and PYCR1 expression in tumor tissues of CRC patients. Collectively, the present study highlighted a previously unrecognized mechanism of B7-H3-mediated rewiring of proline metabolism through increased expression of PYCR1 in CRC cells. These findings suggested that B7-H3 may serve as a novel prognostic factor and a promising therapeutic target for CRC.
\end{abstract}

Keywords: B7-H3; PYCR1; Proline metabolism; Colorectal cancer; Correlation; Prognosis

\section{Abbreviations}

CRC: Colorectal Cancer; B7-H3: B7 Homolog 3; PYCR1: Pyrroline-5-Carboxylate Reductase 1

\section{Introduction}

Colorectal Cancer (CRC) represents the third most frequently diagnosed malignant neoplasm of the gastrointestinal tract and the fourth leading cause of cancer-associated mortality amongst all malignancies worldwide. Recurrence and metastasis remain the primary cause of death in CRC patients and represent a clinical challenge. Therefore, it is highly desirable to understand the molecular mechanisms underlying CRC progression and identify novel prognostic biomarkers and therapeutics for CRC.

B7 Homolog 3 (B7-H3), an essential immune checkpoint member of the B7 and CD28 families, shares 20\% 27\% amino acid homology with other members of the B7 family [1]. B7-H3 is identified to be highly expressed in various tumor tissues and tumor cell lines but low in normal tissues and cells, and its expression in cancer is closely associated with poor prognosis of patients. Limet et al. proposed that immunoregulatory protein $\mathrm{B} 7-\mathrm{H} 3$ affects glucose metabolism in cancer cells mediated by HIF-1A [2]. Liu et al. revealed that B7$\mathrm{H} 3$ was highly expressed in CRC cells and facilitated the migration and invasion of CRC cells [3]. Although these studies have revealed multiple functions of B7-H3 in CRC, the function of B7-H3 in CRC metabolism and the mechanism of promoting tumor progression remain elusive.

Proline, a non-essential amino acid with low molecular weight and highly soluble in water, plays a protective role against oxidative stress and redox homeostasis [4]. Proline is synthesized from glutamate by two enzymes: $\Delta 1$-pyrroline-5-carboxylate synthetase (P5CS) and P5C reductase (P5CR) [5]. In the human, P5CRs is also called as PYCR, and three human PYCR isoenzymes have been identified: Pyrroline-5carboxylate reductase 1 (PYCR1), Pyrroline-5-carboxylate reductase 2 (PYCR2), and Pyrroline-5-carboxylate reductase-like (PYCRL). P5CRs are localized in mitochondria, where the bulk of metabolic reactions take place to support cellular functions [6]. Nisebita et al. revealed that proline consumption and expression of proline synthase were closely associated with clonogenic and tumorigenic potential; besides, inhibition of proline synthase diminished clonogenic and tumorigenic potential [7]. Elia et al. demonstrated that proline catabolism supported the growth of breast cancer cells, which was related to tumor metastasis in vivo [8]. Moreover, Liu et al. found that MYC promoted mRNA expression and protein levels of key enzymes involved in proline synthesis, which can promote cell growth and energy production [9]. Together, these studies indicated that proline plays a crucial role in tumor cell growth.

Although multiple studies have suggested that $\mathrm{B} 7-\mathrm{H} 3$ promotes tumor cell growth and proliferation in CRC, the relationship between B7-H3 and proline metabolism and the exact mechanism of B7-H3 remains largely unknown in CRC. In this study, we found that B7- 
$\mathrm{H} 3$ exhibits a positive relationship with PYCR1, and B7-H3 enhances proline metabolism by up-regulating the expression of PYCR1, which in turn promotes the proliferation and metastasis of CRC cells. The present study's findings reveal a previously unrecognized mechanism by which $\mathrm{B} 7-\mathrm{H} 3$ is involved in proline metabolism and tumor progression in CRC.

\section{Materials and Methods}

\section{Cell lentivirus infection, cell transfection, and cell culture}

The two human CRC cell lines, SW480 and Caco-2 cell lines with different expression levels of B7-H3 were obtained from American Type Culture Collection (ATCC; Manassas, VA, USA). The stable B7-H3-overexpressing SW480 (SW480-B7) cells were generated by transfection with overexpression plasmid, and the stable knockdown of B7-H3 in Caco-2 cells were generated with a B7-H3 shRNA (Caco-2-shB7). SW480-NC and Caco-2-shNC were generated by transfection with a lentivirus carrying a negative control.

For PYCR1 gene knockdown, human PYCR1 siRNA-1, human PYCR1 siRNA-2, human PYCR1 siRNA-3, and their control siRNAs were procured from GenePharma Co., Ltd (Suzhou, China). SW480-B7 and Caco-2-shB7 cells were transfected with siRNAs using Lipofectamine $8000^{\mathrm{TM}}$ Transfection Reagent (Beyotime Institute of Biotechnology, Shanghai, China) following the manufacturer's instructions. The signaling pathway inhibitors used included PI3K inhibitor (LY294002), AKT inhibitor (MK2206). Transfection efficiency was determined by Real-time quantitative PCR (qRT-PCR) and Western blot assay.

Cells were cultured in Dulbecco's modified Eagle medium (DMEM; HyClone, SH30022.01) supplemented with 10\% fetal bovine serum (FBS; Clark Bioscience, Houston, TX, United States ) and $1 \%$ penicillin-streptomycin at $37^{\circ} \mathrm{C}$ in a humidified atmosphere with $5 \% \mathrm{CO}_{2}$.

\section{RNA isolation and RT-qPCR assays}

Total RNA was extracted from the cultured cells using Trizol reagent (Invitrogen, Carlsbad, CA) according to the manufacturer's instructions. For reverse transcription, cDNA was synthesized using the PrimeScript RT-PCR kit (Takara, Japan). The expression level of B7-H3 was determined using the QuantinovATM SYBR Green PCR kit (Qiagen, Hilden, Germany) on an ABI 7500 Realtime PCR system (Applied Biosystems, Foster City, CA, United States). $\beta$-actin was used as endogenous control and the relative expression level of B7$\mathrm{H} 3$ as fold change was calculated using the $2-\Delta \Delta \mathrm{Ct}$ method. Primer sequences used in this experiment were listed in Table 1 . All reactions were performed in triplicate and were repeated thrice. The qRTPCR cycle profile was performed at $95^{\circ} \mathrm{C}$ for $2 \mathrm{~min}$ to activate DNA polymerase, followed by 40 cycles of denaturation at $95^{\circ} \mathrm{C}$ for $5 \mathrm{sec}$ and annealing at $60^{\circ} \mathrm{C}$ for $10 \mathrm{sec}$.

\section{Protein extraction and Western blot analysis}

Total protein was extracted with RIPA lysis buffer supplemented with protease inhibitor, a phosphatase inhibitor, and $100 \mathrm{mmol} / \mathrm{L}$ PMSF (Keygen Biotech, China) on ice for 25min. Protein quantification was performed using the BCA Protein Analysis kit (Beyotime Institute of Biotechnology, Shanghai, China) and adjusted to the same concentration of protein. An equivalent amount of the protein was separated on $10 \%$ SDS-PAGE and then transferred to a Polyvinylidene Fluoride (PVDF) membrane (Merck Millipore, Darmstadt, Germany). The membranes were blocked with 5\% skimmed milk for $1 \mathrm{~h}$ at room temperature. Then, membranes were incubated overnight with the following primary antibodies: mouse anti-human B7-H3 mAb (1:1000, ptoteintech), rabbit anti-human PYCR1 pAb (1:1000, ptoteintech) and mouse anti-human $\beta$-actin $\mathrm{mAb}$ (Beyotime, Nantong, China). After three washes with TBST for 10 minutes each, membranes were incubated with the corresponding goat versus mouse or goat versus rabbit secondary antibodies at room temperature for 1 hour. After washing with TBST, the membrane was treated with enhanced chemiluminescence (ECL) detection reagent (ABSIN, Shanghai, China). The immunoreactive bands were visualized using the Chemidoc ${ }^{\mathrm{TM}} \mathrm{XRS}+$ detection system and the Image $\mathrm{Lab}^{\mathrm{TM}}$ software (Bio-Rad, Hercules, CA, USA). Quantity One (Bio-Rad, Hercules, CA, USA) was used for quantitative analysis.

\section{Proline and glutamic acid yield determination}

Cells were seeded onto a six-well plate and cultured in a serumfree medium. After 24 hours of culture, the supernatant of the cells was collected in a sterile tube. The precipitate was removed by centrifugation and stored at $-20^{\circ} \mathrm{C}$ until further use. The production of proline and glutamate in the culture medium was determined by the Proline (PRO) assay kit (ml107611, mlbio) and human glutamate (Glu) assay kit (ml038308, mlbio), and the absorbance was measured at $520 \mathrm{~nm}$, and $450 \mathrm{~nm}$ with a microplate analyzer, respectively, and these values were normalized to the total protein concentration.

\section{Colony formation, EDU and proliferation assays}

For the colony formation assay, 800 cells per well were seeded into 6-well plates. Following 2-3 weeks of incubation, colonies were washed with PBS, fixed with anhydrous methanol, and stained with $0.5 \%$ crystal violet for $30 \mathrm{~min}$, dried and observed under a microscope.

Cell proliferation ability was assessed using the Cell Counting Kit8 (CCK8 kit; SolarBio, China) assay according to the manufacturer's protocol. In brief, cells were seeded into 96-well plates at a density of 2000 cells per well. After a $24 \mathrm{~h}$ culture, CCK8 reagent was added to each well and incubated for $90 \mathrm{~min}$. Finally, the absorbance at $450 \mathrm{~nm}$ wavelength was measured at $0,24,48,72$, and 96 hours, respectively, after each medium replacement. Each experiment was repeated three times independently, and a growth curve was plotted.

EdU labeling was performed to assess cells' proliferation capacity using the EdU Proliferation Kit (RIBOBIO) according to the manufacturer's instructions. Briefly, 8000 cells per well were seeded in a 96-well plate and incubated for $24 \mathrm{~h}$. Then, cells were fixed with anhydrous methanol for $30 \mathrm{~min}$ and treated with $0.5 \%$ Triton X-100 for $10 \mathrm{~min}$ for permeability. Then, the Apollo reaction cocktail was added and incubated at room temperature for $30 \mathrm{~min}$, followed by staining with Hoechst 33342 for $30 \mathrm{~min}$. After a brief wash with PBS, EdU-stained cells were visualized under Nikon Inverted Research Microscope at 10X and 20X magnification.

\section{Invasion and migration assays}

Transwell assay was used to assess cell invasion ability. Briefly, $5 \times 10^{4}$ cells per well were seeded into 24 -well plates, and $100 \mu \mathrm{L}$ serum-free medium was added to the upper membrane, and $10 \%$ FBS -containing medium was added to the lower chamber. After 24 hours, the cells remaining on the upper membrane were carefully 
Table 1: Primers for reverse transcription-quantitative polymerase chain reaction.

\begin{tabular}{|c|c|c|}
\hline Gene & Forward sequence (5'-3') & Reverse sequence (5'-3') \\
\hline B7-H3 & AGCACTGTGGTTCTGCCTCACA & CACCAGCTGTTTGGTATCTGTCAG \\
\hline PYCR1 & TGGCTGCCCACAAGATAATGG & CGTGACGGCATCAATCAGGT \\
\hline PYCR2 & TCCTGTCGGCTCACAAGATAA & AGGGGATGATATGTGGCTTCA \\
\hline PYCRL & CGTGTGTGCATTCTCCGAG & GGAGTCCATAGATGGTGGTGC \\
\hline$\beta$-actin & CATGTACGTTGCTATCCAGGC & CTCCTTAATGTCACGCACGAT \\
\hline
\end{tabular}

wiped off with a cotton swab, while the cells that had invaded through the membrane were fixed with anhydrous methanol and stained with $0.5 \%$ crystal violet for $20 \mathrm{~min}$ at room temperature. Cells were then counted in five randomly selected fields (at $\times 100$ magnification) under a microscope. All experiments were repeated independently three times.

For migration assay, $5 \times 10^{4}$ cells were seeded to the upper chamber of each well (coated with $20 \mu \mathrm{L}$ Matrigel) then, FBS-containing complete medium was added to the lower chamber. After incubation for $24 \mathrm{~h}$, cells that migrated to the lower membrane of the chamber were fixed with anhydrous methanol and stained with $0.5 \%$ crystal violet for $20 \mathrm{~min}$ at room temperature. Cells were then counted in five randomly selected fields (at $\times 100$ magnification) under an inverted microscope. All experiments were repeated independently three times.

Cells were cultured in a 6-well plate at a density of $1 \times 10^{5}$ per well. After incubation for 24 hours, the monolayers were scratched with a 10-10- $\mu \mathrm{L}$ tip. Following washing with PBS, cellular migration toward the scratched area was captured under a microscope at 0,24 , and 48 hours, respectively.

\section{Patients and tissue sample}

The medical records of patients who were diagnosed and underwent CRC surgical resection at the Affiliated Hospital of Jiangnan University between June 2008 and December 2011 were retrieved. A total of 206 formalin-fixed paraffin-embedded tissue samples from CRC patients were included. These patients did not receive preoperative radiotherapy or chemotherapy, and the clinical data and pathological tissue were intact. This study was approved by the Medical Ethics Committee of the Affiliated Hospital of Jiangnan University, and written informed consent was obtained from each patient. To obtain patient survival data, all patients were followed over the telephone until October 31,2017, with a median followup time of 79 months (range 6-114 months). Two experienced pathologists examined hematoxylin and eosin (H\&E) stained slides to confirm tumor tissue and adjacent normal tissue. The corresponding spots on the tissue block were marked for a correct tissue core punch, which was cut into $4 \mu \mathrm{m}$ thick continuous sections and affixed to antidewaxing glass slides.

\section{Immunochemistry}

The tissue sections were deparaffinized in xylene and rehydrated in a graded series of ethanol. Antigen retrieval was performed by heating the tissue sections at $100^{\circ} \mathrm{C}$ in sodium citrate buffer in a microwave oven for 30 minutes. The endogenous peroxidase activity was blocked by $3 \%$ hydrogen peroxide for 10 minutes. Subsequently, the non-specific protein was blocked by incubation with 5\% skim milk at room temperature for $30 \mathrm{~min}$, followed by incubation with the following primary antibodies: mouse anti-human B7-H3 monoclonal antibody (1:200, Santa Cruz, Dallas, TX, United States) and rabbit antihuman PYCR1 Polyclonal antibody (1:100, proteintech) overnight at $4^{\circ} \mathrm{C}$. Then, sections were washed with PBS and incubated at room temperature with secondary antibody (1:1; GK600710, Genetech, Shanghai, China) for one hour. The immunostaining was carried out by staining with 3, 3'-diaminobenzidine chromogen (DAB substrate; 1:1; GK600710, Genetech, Shanghai, China) and counterstained with hematoxylin for $60 \mathrm{~s}$ at room temperature, dehydrated and mounted, and the sections were examined under a microscope.

\section{Evaluation of immunochemistry staining}

The sections were examined in a blinded manner by two independent pathologists. Both the intensity and extent of immunological staining of B7-H3 and PYCR1 were analyzed semiquantitatively and scored according to the percentage of positively stained tumor cells and the staining intensity. The percentage of positively stained cells was scored as follows: $\leq 5 \%$ positive cells (score of 0 ), $6 \%$ to $25 \%$ positive cells (score of 1 ), $26 \%$ to $50 \%$ positive cells (score of 2), $51 \%$ to $75 \%$ positive cells (score of 3 ) and $\geq 76 \%$ positive cells (score of 4). The staining intensity was scored as follows: 0 (no staining), 1 (weakly positive), 2 (moderately positive), and 3 (strongly positive). The immunoreactivity score for each specimen was multiplied by the percentage of positively stained cell score and the intensity score. According to the total score, we defined $0 \sim 3$ as low expression and $4 \sim 12$ as high expression.

\section{Statistical analysis}

Statistical analysis was performed with IBM SPSS Statistics software (22.0; IBM, Chicago, IL USA. The association between the clinicopathological data and the protein expression levels of PYCR1 and $\mathrm{B} 7-\mathrm{H} 3$ was assessed by the chi-square test. The overall survival was calculated using the Kaplan-Meier curve and compared using the log-rank test. The proportional hazard model method was used to perform the univariate and multivariate regression analyses and compared using Cox proportional risk regression analysis. A twotailed p-value of $<0.05$ was considered statistically significant.

\section{Results}

\section{B7-H3 promotes proline metabolism in CRC cells}

In order to determine the expression of B7-H3 in CRC cells, the expression of B7-H3 was analyzed in six intestinal cancer cells by Western blot assay, including HCT-8, Caco-2, SW480, Lovo, DLD1, and HCT-116 (Figure 1A). The results revealed that the expression of B7-H3 was markedly higher in Caco-2 cells and lower in SW480 cells. Therefore, four stable transgenic strains expressing high and low B7-H3 were established (SW480-NC, SW480-B7; Caco-2-shNC, 
A

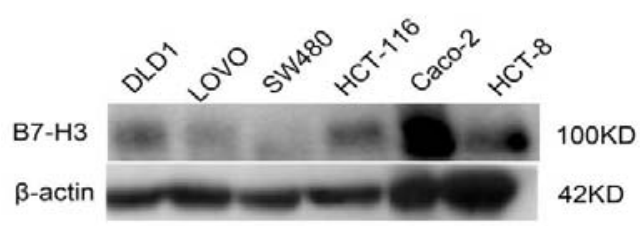

C

SW480

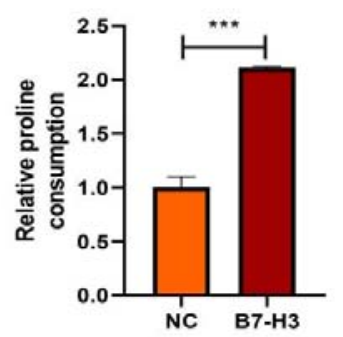

D

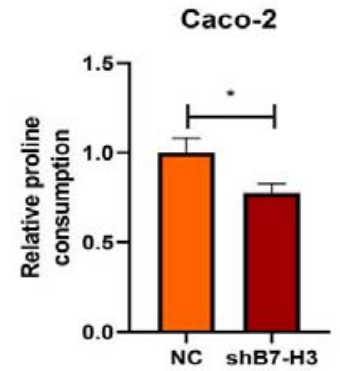

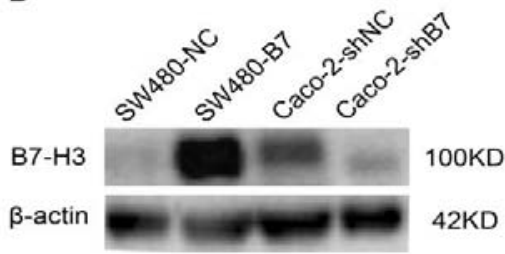

E

$\mathbf{F}$

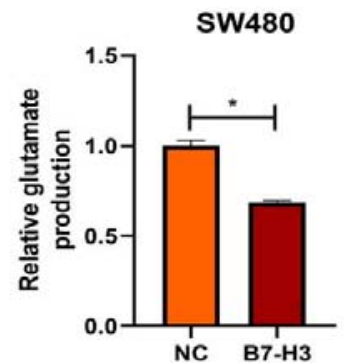

Figure 1: B7-H3 promotes proline metabolism in CRC cells. A) The expression of B7-H3 was higher in Caco-2 cells and lower in SW480 cells. B) SW480 cells with overexpression of B7-H3 and Caco-2 cells with B7-H3 knockdown were detected by Western blot assay. C) Proline consumption of SW480-B7 cells. D) Proline consumption of Caco-2-shB7 cells. E) Glutamate production of SW480-B7 cells. F) Glutamate production of Caco-2-shB7 cells. ${ }^{\star} \mathrm{P}<0.05$; ${ }^{\star *} \mathrm{P}<0.01$; ${ }^{\star *} \mathrm{P}<0.001$.

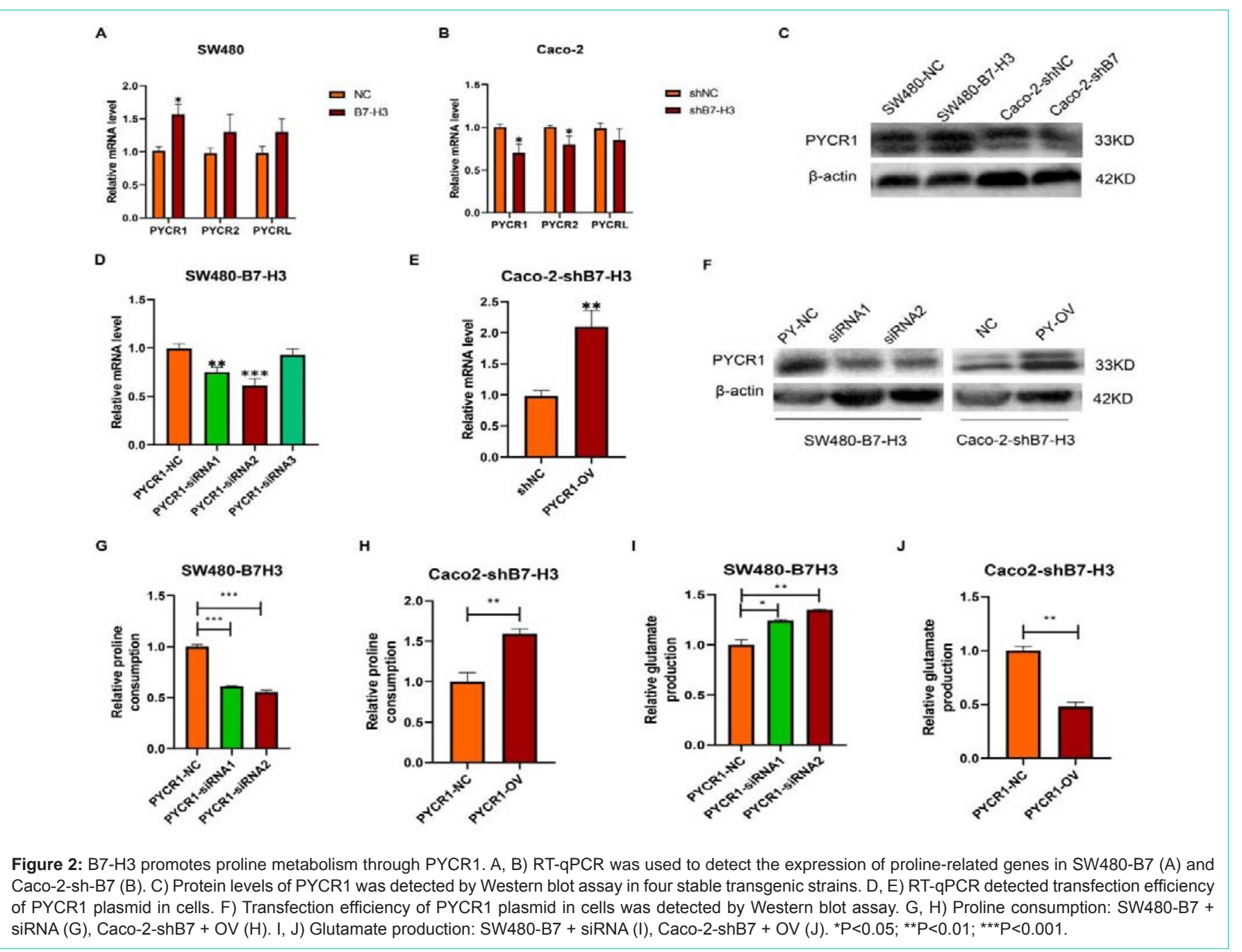


Caco-2-shB7) (Figure 1B). The consumption of proline in SW480-B7 cells with overexpression of $\mathrm{B} 7-\mathrm{H} 3$ was significantly higher compared with the control group (Figure 1C), and the production of glutamate was lower (Figure 1E). Similarly, Caco-2 cells with B7-H3 knockdown significantly reduced proline consumption (Figure 1D), and significantly increased glutamate production compared with the control group (Figure 1F).

\section{B7-H3 promotes proline metabolism through PYCR1}

To investigate the effect of $\mathrm{B} 7-\mathrm{H} 3$ on proline metabolism in CRC cells, we selected four cell lines, including SW480-NC, SW480-B7, Caco-2-shNC, and Caco-2-shB7, and analyzed the expression of key genes involved in proline metabolism using qRT-PCR, including PYCR1, PYCR2, and PYCRL. The results showed that compared with the SW480-NC group, the expression of all three genes in SW480-B7 cells were increased (Figure 2A). Compared with the Caco-2-shNC group, the expression of all three genes in Caco-2-shB7 cells decreased (Figure 2B), but the change of PYCR1 was the most obvious, so PYCR1 was selected as the target gene. In addition, Western blot analysis revealed that PYCR1 was highly expressed in SW480-B7 cells, while it was significantly decreased in Caco-2-shB7 cells compared with the control group (Figure 2C). Therefore, our results indicated that high $\mathrm{B} 7-\mathrm{H} 3$ positively regulated the expression of PYCR1. When PYCR1 siRNA down-regulated PYCR1 in SW480-B7 cells, proline consumption decreased and glutamate production increased (Figure 2G and 2H). After Caco2-shB7 was overexpressed with PYCR1, overexpression of PYCR1 abolished the inhibition of proline metabolism by under expression of B7-H3 (Figure 2I and 2J). These results suggested that $\mathrm{B} 7-\mathrm{H} 3$-induced proline metabolism was dependent on PYCR1.

\section{B7-H3 induces the proliferation of CRC cells by PYCR1}

Colony formation experiments revealed that the number of colonies of SW480-B7 cells with B7-H3 overexpression was higher, while the number of colonies of Caco-2-shB7 cells with B7-H3 knockdown was lower, indicating that $\mathrm{B} 7-\mathrm{H} 3$ overexpression could promote clonogenic ability (Figure $3 \mathrm{~A}$ and $3 \mathrm{~B}$ ). However, when PYCR1-siRNA inhibited the SW480-B7 cells, the number of cell clones decreased, and the number of cell clones increased significantly after the overexpression of PYCR1 by Caco2-shB7-H3, suggesting that $\mathrm{B} 7-\mathrm{H} 3$ may regulate the clonogenic ability of $\mathrm{CRC}$ cells by mediating PYCR1 (Figure 3C and 3D).

In CCK8 experiments on CRC cells, SW480-B7 cell line with B7-H3 overexpression promoted cell proliferation, while Caco2-shB7 cell line with B7-H3 knockdown decreased proliferation (Figure $3 \mathrm{E}$ and $3 \mathrm{~F}$ ), indicating that $\mathrm{B} 7-\mathrm{H} 3$ can positively regulate the proliferation of CRC cells. Interestingly, when the expression of PYCR1 was inhibited, the proliferation rate of SW480-B7 cells also decreased, while the proliferation ability of Caco-2-shB7 cells increased following the overexpression of PYCR1 (Figure 3G and $3 \mathrm{H})$.

EDU staining revealed that the higher $\mathrm{B} 7-\mathrm{H} 3$ expression and the more number cell colonies (Figure 3I). Inhibition of PYCR1 expression significantly reduced cell proliferation (Figure 3J). These experiments suggested that the effect of $\mathrm{B} 7-\mathrm{H} 3$ on the proliferative ability of CRC cells might be through the regulation of PYCR1 expression.

\section{B7-H3 supports the invasion and migration of CRC cells through PYCR1}

Transwell assay was performed to investigate whether B7-H3 was involved in CRC cell invasion through PYCR1. We observed that in SW480-B7 cell lines, the number of cell invasions in the B7$\mathrm{H} 3$ overexpressed group was higher than the control group, but the number of cell invasions in the two PYCR1-siRNA groups was significantly reduced compared with the NC group (Figure 4A and 4C). Similarly, in Caco-2-shB7 cell lines, the number of invading cells in the B7-H3 knockdown group was less than that in the NC group, while the number of invading cells in the overexpression group of PYCR1 was increased (Figure 4B and 4D). These results suggested that B7-H3 supports CRC cell invasion through PYCR1

We investigated the effect of $\mathrm{B} 7-\mathrm{H} 3$ on CRC cell migration via PYCR1 using a scratch wound-healing assay. The results revealed that the migration ability of SW480-B7 cells was significantly increased compared with the NC group, but the migration ability of SW480$\mathrm{B} 7 \mathrm{H} 3$ cells was decreased after the inhibition of PYCR1 (Figure $4 \mathrm{E}$ and $4 \mathrm{G})$. Similarly, the migration ability of Caco2-shB7-H3 cells was significantly decreased compared with the NC group, but the migration ability was increased in the Caco2-shB7-H3 cells overexpressing PYCR1 (Figure $4 \mathrm{~F}$ and $4 \mathrm{H}$ ). The results indicated that B7-H3 promoted the migration of CRC cells through PYCR1.

\section{The expression of B7-H3 was associated with PYCR1}

Immunohistochemical staining was used to detect the expression levels of B7-H3 and PYCR1 in 205 CRC tissues. The results revealed that $\mathrm{B} 7-\mathrm{H} 3$ staining was predominantly localized in the cell membrane and cytoplasm (Figure 5C), while PYCR1 was mainly localized in the cytoplasm (Figure 5D). According to the staining intensity of the two molecules, the samples were divided into low expression and high expression groups. The positive rates of $\mathrm{B} 7-\mathrm{H} 3$ and PYCR1 were $57.1 \%$ and $50.2 \%$, respectively. $\mathrm{B} 7-\mathrm{H} 3$ was significantly associated with age $(\mathrm{P}=0.029)$, lymph node metastasis $(\mathrm{P}=0.025)$, TNM stage $(\mathrm{P}=0.035)$, and vascular invasion $(\mathrm{P}=0.042)$, while the expression of PYCR1 was significantly associated with gender $(\mathrm{p}=0.016)$, and lymph node metastasis $(\mathrm{P}=0.036)$, and TNM stage $(\mathrm{P}=0.036)$ (Table 1$)$. Meanwhile, among the 205 cases of CRC, 68 cases (33.2\%) exhibited high expression of B7-H3 and PYCR1, and 53 cases (25.9\%) exhibited low expression of B7-H3 and PYCR1. Spearmen correlation analysis showed that $\mathrm{B} 7-\mathrm{H} 3$ was positively correlated with PYCR1 expression (correlation coefficient was 0.5987; P <0.0001) (Figure 5E).

\section{High expression of $\mathrm{B} 7 \mathrm{H} 3$ and PYCR1 associates with poor prognosis of patients with CRC}

The survival analysis revealed that patients with high $\mathrm{B} 7-\mathrm{H} 3$ expression exhibited a significantly worse prognosis compared to those with low B7-H3 expression ( $\mathrm{P}=0.033$; Figure 5F). Similarly, patients with high PYCR1 expression had a significantly worse prognosis than those with low PYCR1 expression ( $\mathrm{P}=0.043$; Figure 5G). In addition, to further explore the combined effect of B7-H3 and PYCR1 on CRC patients, we grouped them, and results indicated that patients with high B7-H3 expression and high PYCR1 expression subgroups had a significantly worse prognosis compared to patients with low B7$\mathrm{H} 3$ and PYCR1 expression $(\mathrm{P}=0.042$; Figure $5 \mathrm{H})$. However, there was 
A
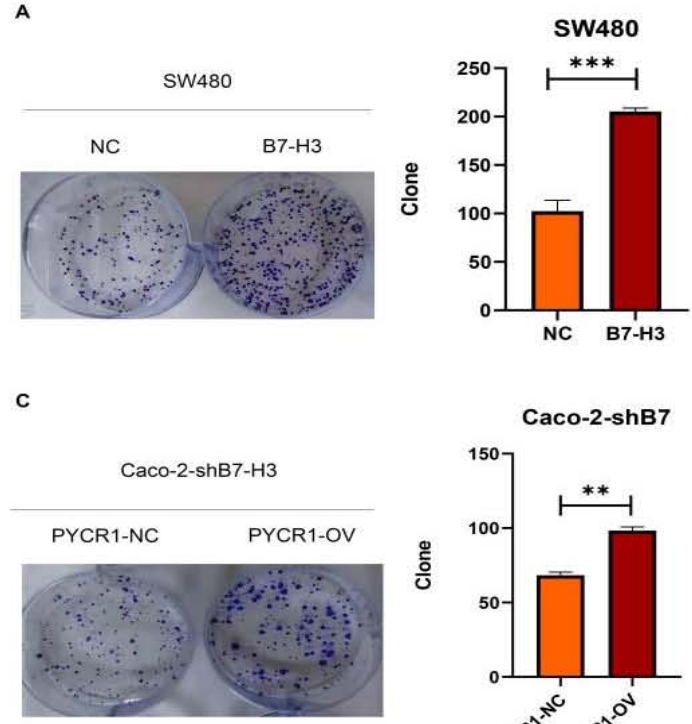

E

SW480

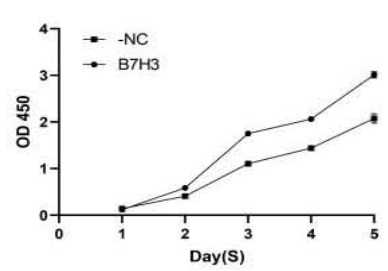

I

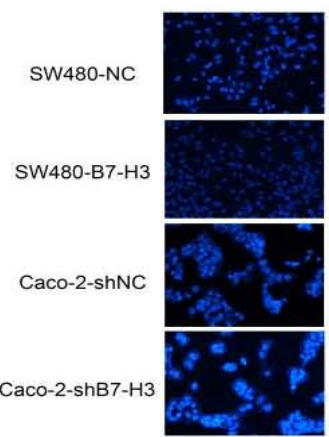

DAPI

SW480

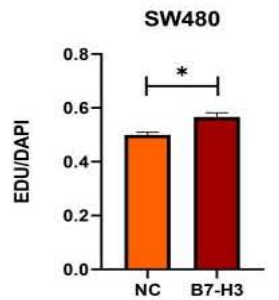

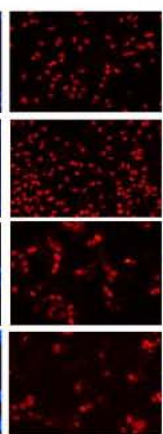

EDU

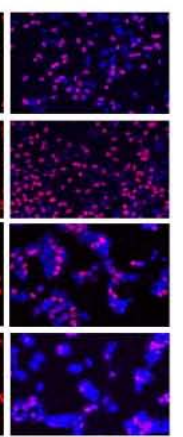

Merge
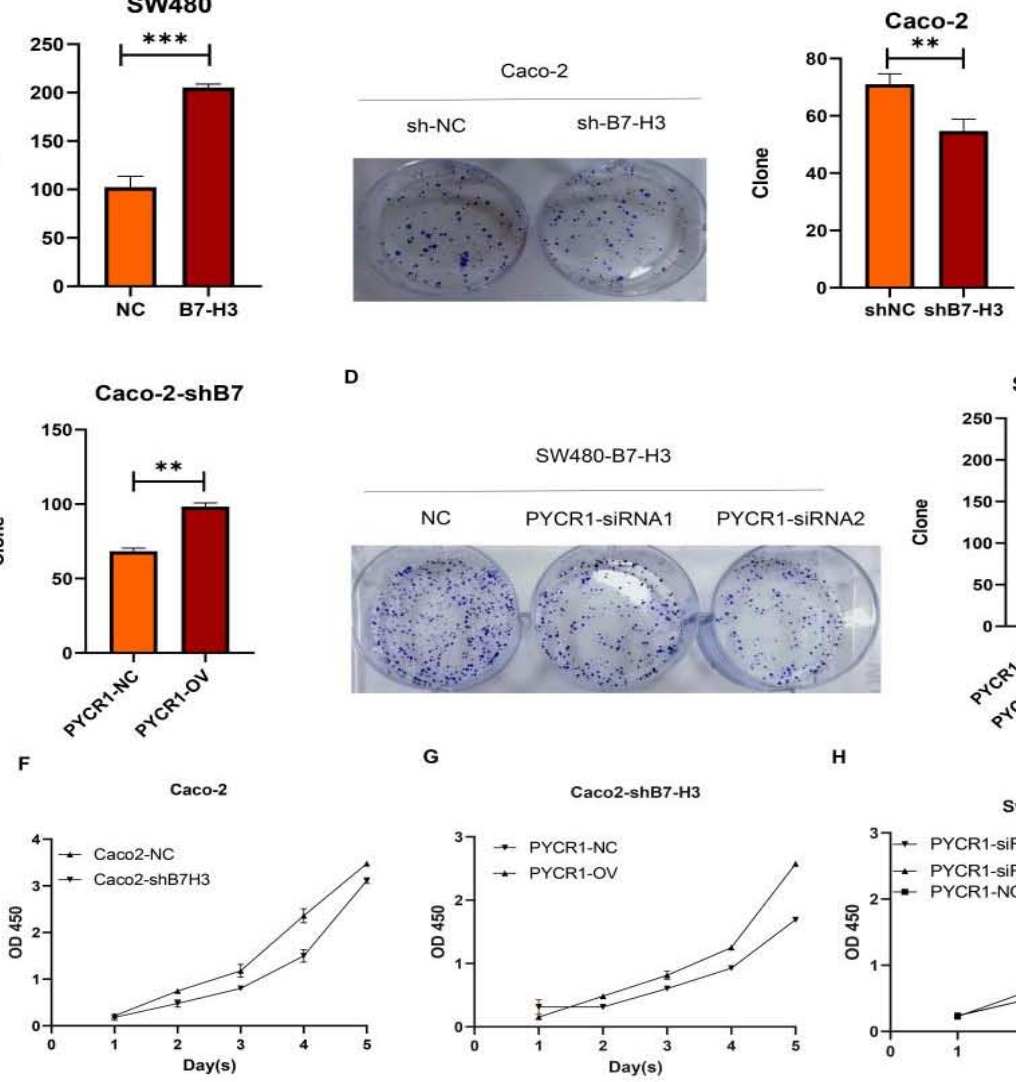

D

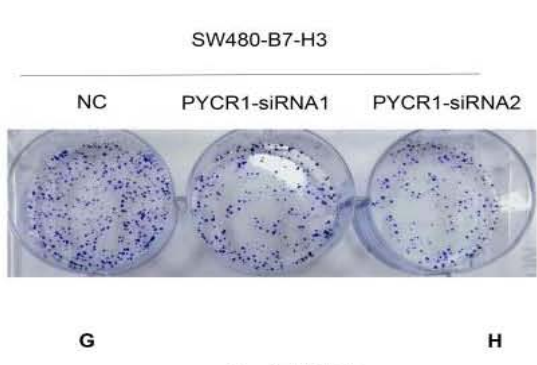

Caco2-shB7-H3
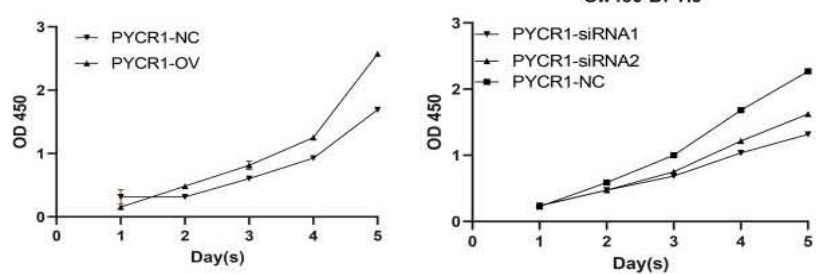

J

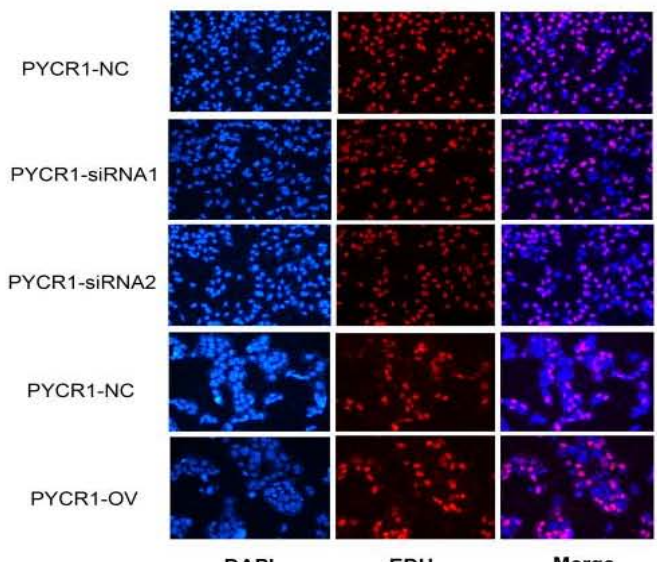

EDU

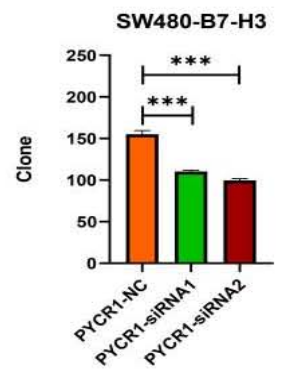

Sw480-B7-H3

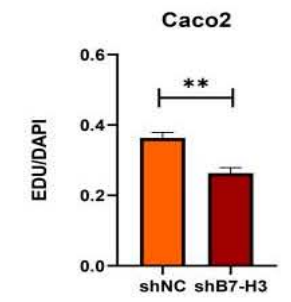

Figure 3: B7-H3 induces the proliferation of CRC cells by PYCR1. A-D) High expression of B7-H3 promoted the number of CRC cells by mediating PYCR1 in Colony formation experiments: SW480-B7 (A), Caco-2-shB7 (B), Caco-2shB7-H3-OV (C), SW480-B7-siRNA (D). E-H) Cell viability was analyzed using the CCK8 kit: SW480-B7 (E), Caco-2-shB7 (F), Caco-2-shB7+OV (G), SW480-B7+siRNA (H). I, J) EDU assay was used to detect cell proliferation. *P<0.05; ** $<0.01$; $\star \star * P<0.001$. 
Table 2: Expression of B7-H3 and PYCR1 in colorectal cancer patients and their correlation with clinicopathological parameters.

\begin{tabular}{|c|c|c|c|c|c|c|c|}
\hline \multirow{2}{*}{ Clinicopathological parameter } & \multirow{2}{*}{ Case (n) } & \multicolumn{2}{|c|}{ B7-H3 expression } & \multirow{2}{*}{$P$ value } & \multicolumn{2}{|c|}{ PYCR1 expression } & \multirow{2}{*}{$P$ value } \\
\hline & & Low & High & & Low & High & \\
\hline Gender & & & & 0.319 & & & 0.016 \\
\hline Male & 117 & 54 & 63 & & 67 & 50 & \\
\hline Female & 88 & 34 & 54 & & 35 & 53 & \\
\hline Age (yrs) & & & & 0.029 & & & 0.47 \\
\hline$<60$ & 76 & 25 & 51 & & 35 & 41 & \\
\hline$\geq 60$ & 129 & 63 & 66 & & 67 & 62 & \\
\hline Tumor location & & & & 0.563 & & & 0.775 \\
\hline Colon & 81 & 37 & 44 & & 39 & 42 & \\
\hline Rectum & 123 & 50 & 73 & & 63 & 60 & \\
\hline Colon cancer site & & & & 0.575 & & & 0.574 \\
\hline Left-sided & 171 & 75 & 96 & & 87 & 84 & \\
\hline Right-sided & 34 & 13 & 21 & & 15 & 19 & \\
\hline Depth of tumor invasion & & & & 0.643 & & & 0.646 \\
\hline $\mathrm{T} 1 / 2$ & 60 & 24 & 36 & & 28 & 32 & \\
\hline T3/4 & 145 & 64 & 81 & & 74 & 71 & \\
\hline Lymph node metastasis & & & & 0.025 & & & 0.036 \\
\hline NO & 107 & 54 & 53 & & 61 & 46 & \\
\hline $\mathrm{N} 1 / 2$ & 98 & 34 & 64 & & 41 & 57 & \\
\hline Distant metastasis & & & & 0.244 & & & 0.815 \\
\hline Yes & 185 & 82 & 103 & & 93 & 92 & \\
\hline No & 20 & 6 & 14 & & 9 & 11 & \\
\hline III/IV & 104 & 37 & 67 & & 44 & 60 & \\
\hline Neural invasion & & & & 0.419 & & & 0.689 \\
\hline Yes & 176 & 78 & 98 & & 89 & 87 & \\
\hline No & 29 & 10 & 19 & & 13 & 16 & \\
\hline Vascular invasion & & & & 0.042 & & & 0.066 \\
\hline Yes & 177 & 81 & 96 & & 93 & 84 & \\
\hline No & 28 & 7 & 21 & & 9 & 19 & \\
\hline Mucinous adenocarcinoma & & & & 1 & & & 0.631 \\
\hline Yes & 186 & 80 & 106 & & 94 & 92 & \\
\hline No & 19 & 8 & 11 & & 8 & 11 & \\
\hline
\end{tabular}

no statistically significant difference in the prognosis of CRC patients in the subgroup with low $\mathrm{B} 7-\mathrm{H} 3$ and PYCR1 expression ( $\mathrm{P}=0.430$, Figure 5I).

Univariate Cox proportional risk model was used to analyze and evaluate the risk factors related to the prognosis of CRC patients. The results revealed that high expression of $\mathrm{B} 7-\mathrm{H} 3$, high expression of PYCR1, depth of tumor invasion, lymph node metastasis, distant metastasis, TNM stage, nerve invasion, and vascular invasion were associated with OS in CRC patients. In addition, multivariate analysis indicated that distant metastasis was an independent prognostic factor for predicting the survival in CRC patients, as shown in Table 3.

\section{Discussion}

B7-H3, a member of the B7 family of immunoregulatory proteins, is aberrantly overexpressed in a variety of cancer and identified to regulate key metabolic enzymes, promoting the high glycolytic potential of cancer cells. Shi et al. proposed that B7-H3 could promote aerobic glycolysis in CRC by mediating HK2 [10]. Studies have shown a correlation of IDH1 and B7-H3 expression with the prognosis of patients with CRC [11]. However, as one of the mechanisms of tumor metabolic reprogramming, the relationship between B7-H3 and proline metabolism in CRC remains to be elucidated. This study found that proline metabolism was enhanced in cells overexpressing $\mathrm{B} 7-\mathrm{H} 3$. In order to explore the regulatory mechanism of B7-H3 and 


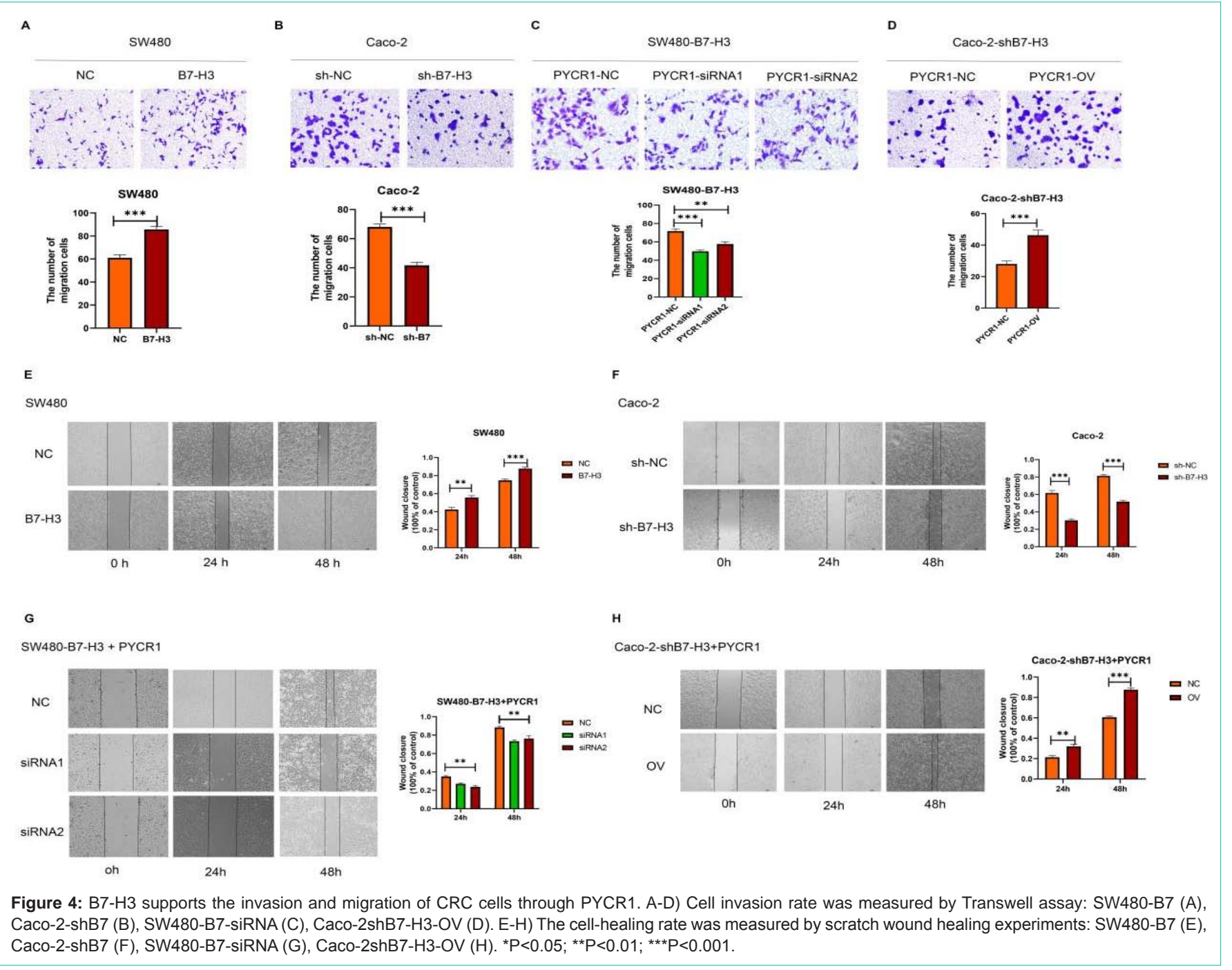

Table 3: Univariate and multivariate of factors associated with overall survival rate.

\begin{tabular}{|c|c|c|c|c|c|c|}
\hline \multirow{2}{*}{ Characteristics } & \multicolumn{3}{|c|}{ Univariate analysis } & \multicolumn{3}{|c|}{ Multivariate analysis } \\
\hline & Hazard ratio & $95 \% \mathrm{Cl}$ & P-Value & Hazard radio & $95 \% \mathrm{Cl}$ & P-Value \\
\hline Gender & 0.954 & $0.609-1.495$ & 0.836 & 0.991 & $0.612-1.604$ & 0.971 \\
\hline Age (yrs) & 1.005 & $0.633-1.598$ & 0.982 & 1.159 & $0.705-1.907$ & 0.56 \\
\hline Tumor location & 0.727 & $0.464-1.140$ & 0.165 & 0.803 & $0.440-1.467$ & 0.476 \\
\hline Colon cancer site & 1.286 & $0.732-2.259$ & 0.382 & 1.27 & $0.613-2.632$ & 0.52 \\
\hline Depth of tumor invasion & 2.699 & $1.458-4.996$ & 0.002 & 1.746 & $0.888-3.432$ & 0.106 \\
\hline Lymph node metastasis & 2.546 & $1.594-4.066$ & 0 & 0.686 & $0.217-2.168$ & 0.521 \\
\hline Distant metastasis & 10.622 & $6.112-18.460$ & 0 & 6.052 & $2.773-13.211$ & 0 \\
\hline TNM stage & 2.998 & $1.839-4.886$ & 0 & 2.482 & $0.728-8.467$ & 0.146 \\
\hline Neural invasion & 3.051 & $1.829-5.091$ & 0 & 1.014 & $0.466-2.204$ & 0.973 \\
\hline Vascular invasion & 2.431 & $1.430-4.132$ & 0.001 & 1.603 & $0.813-3.162$ & 0.173 \\
\hline Mucinous adenocarcinoma & 0.947 & $0.435-2.060$ & 0.891 & 0.572 & $0.246-1.332$ & 0.195 \\
\hline $\mathrm{B} 7 \mathrm{H} 3$ & 2.113 & 1.2903 .460 & 0.003 & 1.487 & $0.857-2.579$ & 0.158 \\
\hline PYCR1 & 1.821 & $1.149-2.887$ & 0.011 & 1.286 & $0.775-2.133$ & 0.33 \\
\hline
\end{tabular}




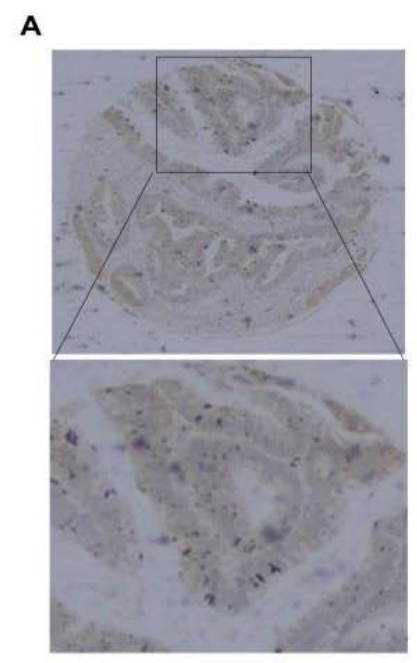

E

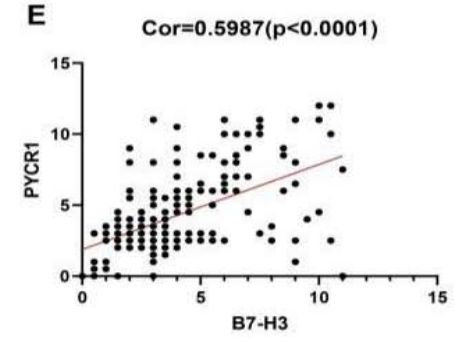

H

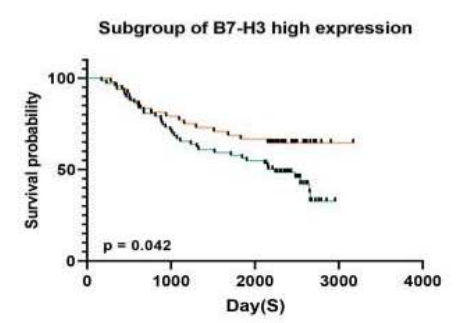

B
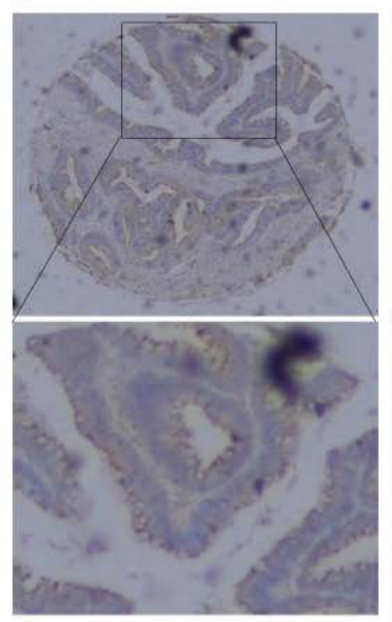

F

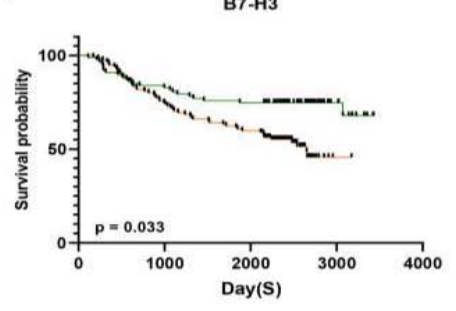

I

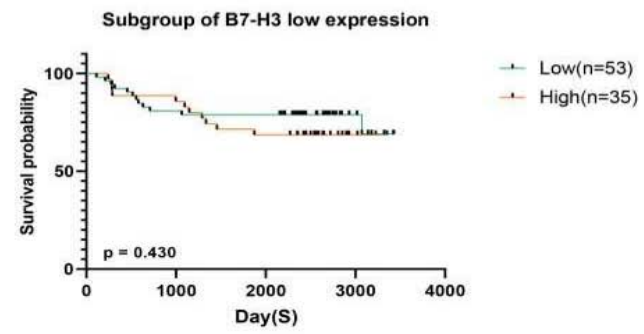

D
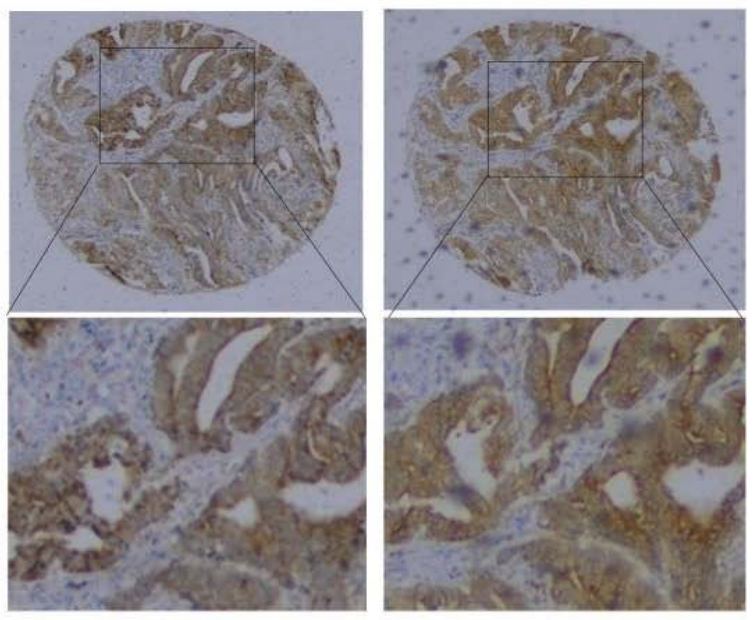

G
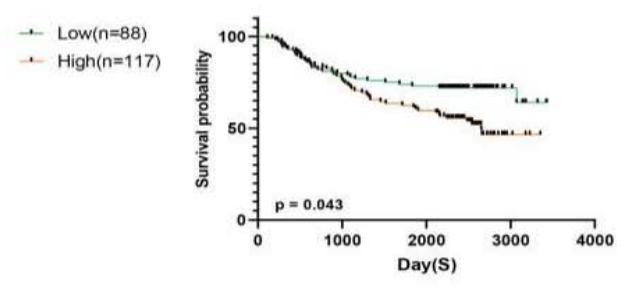

- $\operatorname{Low}(n=102)$

- $\operatorname{High}(n=103)$

Figure 5: B7-H3 associates with PYCR1 and overexpression predicts a worse prognosis in CRC patients. A-D) IHC image of B7-H3 and PYCR1: low expression of B7-H3 (A), low expression of PYCR1 (B), high expression of B7-H3 (C), high expression of PYCR1 (D). E) Correlation analysis of the staining index of expression levels of B7-H3 and HK2 protein. F) Patients with low expression of B7-H3 vs. high expression of B7-H3. G) Patients with low expression of PYCR1 vs. high expression of PYCR1. H) Subgroup of patients with high expression of B7-H3 and low expression of PYCR1 vs. high expression of PYCR1. I) Subgroup of patients with low expression of B7-H3 and high expression of PYCR1 vs. low expression of PYCR1.

proline metabolism, we selected three isozymes of PYCR, including PYCR1, PYCR2, and PYCRL. The qRT-PCR results indicated that the expression of PYCR1 mRNA was significantly associated with that of B7-H3.

PYCR1 is a mitochondrial enzyme involved in the do novo biosynthesis of the proline. PYCR1 encoded by the gene PYCR1, localized on chromosome 17q25.3, comprises 319 amino acids [12]. The protein consists of five homodimers that form a circular groove, which is the binding site of the cofactor and substrate [13]. PYCR1 participates in proline metabolism through catalytic reduction of 5C to proline and production of NADP+, which is closely linked to the TCA urea cycle and pentose phosphate pathway [14]. Recent studies have also demonstrated that PYCR1 is closely related to the occurrence and development of malignant tumors, which mainly confers a potent influence on the growth and development of tumor cells by affecting the metabolism of proline. Gao et al. demonstrated that PYCR1 gene knockdown could inhibit the proliferation, migration, and invasion of lung adenocarcinoma by modulating the Jak/Stat signaling pathway [15]. Expression of PYCR1 has also been identified to promote papillary renal cell carcinoma through the Akt/mTOR pathway [16]. These findings suggest that PYCR1 might be an important prognostic biomarker and therapeutic target for cancer. When PYCR1 in SW480-B7 cells overexpressing B7-H3 was inhibited, the proline metabolic capacity originally activated by B7-H3 was reduced, while PYCR1 in Caco-2-shB7 cells with B7-H3 knockdown was overexpressed, the proline metabolic capacity was also enhanced, suggesting that $\mathrm{B} 7-\mathrm{H} 3$ can mediate PYCR1 to regulate proline metabolism.

An increasing number of studies have indicated that B7-H3 and PYCR1 are related to the progression of tumor cells. The present 
study also evidenced that the overexpression of B7-H3 in CRC cells can promote cell proliferation, invasion, and migration. In addition, when the expression of PYCR1 in SW480-B7 cells was inhibited, the growth and migration ability of CRC cells were reduced. However, when the expression of PYCR1 in Caco-2 cells was overexpressed, the proliferation, invasion and migration ability of CRC cells were significantly improved. These results suggested that B7-H3 affected the proliferation, invasion, and migration of CRC cells by regulating the expression of PYCR1. In order to verify this result and explore the clinical significance of B7-H3 and PYCR1, pathological tissues of 205 CRC patients were collected. Based on the immunohistochemical staining analysis, the relationship between B7-H3 and PYCR1 and clinicopathological parameters was analyzed. B7-H3 and PYCR1 were significantly associated with lymph node metastasis and TNM stage. And the two molecules have expression correlation. More importantly, overexpression of both molecules was associated with a worse prognosis, suggesting that the combined expression of $\mathrm{B} 7-\mathrm{H} 3$ and PYCR1 could be a promising prognostic marker.

To sum up, we investigated the role of $\mathrm{B} 7-\mathrm{H} 3$ in $\mathrm{CRC}$ proline metabolism, illuminated the important molecular mechanisms of the development of resistance in CRC. We found that $\mathrm{B} 7-\mathrm{H} 3$ promotes proline metabolism by regulating the expression of PYCR1 in CRC cells. Furthermore, B7-H3 mediated proline can enhance the proliferation and migration of CRC cells. To the best of our knowledge, this study for the first time revealed the relationship between B7-H3 and proline metabolism and illuminated a previously unrecognized mechanism of B7-H3-mediated rewiring of proline metabolism through increased expression of PYCR1 in CRC cells, suggesting that B7-H3 might serve as a potentially promising therapeutic target for CRC.

\section{Conclusion}

In conclusion, our results suggest that overexpression of $\mathrm{B} 7-\mathrm{H} 3$ and PYCR1 is associated with poor prognosis in CRC patients, and that $\mathrm{B} 7-\mathrm{H} 3$ promotes the proliferation and migration of colorectal cancer cells by regulating the role of PYCR1 in proline metabolism. These results not only indicate the potential of $\mathrm{B} 7-\mathrm{H} 3$ as a prognostic target, but also provide a basis for the study of the mechanism of B7H3 in tumor metabolism.

\section{Declaration}

Funding Statement: This work was supported by Wuxi social development science and technology demonstration project (Demonstration study on the application of immunomolecular typing of colorectal cancer based on intestinal microflora analysis, N20201005), Postgraduate Research \& Practice Innovation Program of Jiangsu Province (KYCX20_1961), Precision Medical Project of Wuxi City (Precision medicine study on the reversal of fluorouracil resistance in colorectal cancer by the targeted delivery of miR-1307 by composite nanocarriers, J202003), Jiangsu Province 333 Highlevel Talents Training Project (Research on prognosis prediction model of gastric cancer based on EBV infestation-related intestinal microorganism analysis BRA2019153).
Author contributions: Yufen J, YM and DH designed the research. Yufen J, TZ and XJ peformed the research. Yufen J and XJ analyzed the data. Yufen J, HL, Rendi W wrote the manuscript. Xinyi $\mathrm{X}$, Xiaoting Wang, Lihua Z, YM, and DH revised the manuscript. All authors contributed to the article and approved the submitted version.

Acknowledgements: This study was reviewed and approved by Affiliated Hospital of Jiangnan University Institutional Review Board.

\section{References}

1. Picarda E, Ohaegbulam KC, Zang X. Molecular Pathways: Targeting B7-H3 (CD276) for Human Cancer Immunotherapy. Clin Cancer Res. 2016; 22: 3425-3431

2. Lim S, Liu H, Madeira da Silva L, Arora R, Liu Z, Phillips JB, et al. Immunoregulatory Protein B7-H3 Reprograms Glucose Metabolism in Cancer Cells by ROS-Mediated Stabilization of HIF1alpha. Cancer Res. 2016; 76: 2231-2242.

3. Liu F, Zhang $\mathrm{T}$, Zou S, Jiang B, Hua D. B7H3 promotes cell migration and invasion through the Jak2/Stat3/MMP9 signaling pathway in colorectal cancer. Mol Med Rep. 2015; 12: 5455-5460.

4. Phang JM, Liu W, Hancock CN, Fischer JW. Proline metabolism and cancer: emerging links to glutamine and collagen. Curr Opin Clin Nutr Metab Care. 2015; 18: 71-77.

5. Pandhare J, Donald SP, Cooper SK, Phang JM. Regulation and function of proline oxidase under nutrient stress. J Cell Biochem. 2009; 107: 759-768.

6. Phang T-H. Salt Tolerance in Soybean. 2008.

7. Sahu N, Dela Cruz D, Gao M, Sandoval W, Haverty PM, Liu J, et al. Proline Starvation Induces Unresolved ER Stress and Hinders mTORC1-Dependent Tumorigenesis. Cell Metab. 2016; 24: 753-761.

8. Elia I, Broekaert D, Christen S, Boon R, Radaelli E, Orth MF, et al. Proline metabolism supports metastasis formation and could be inhibited to selectively target metastasizing cancer cells. Nat Commun. 2017; 8: 15267.

9. Liu W, Hancock CN, Fischer JW, Harman M, Phang JM. Proline biosynthesis augments tumor cell growth and aerobic glycolysis: involvement of pyridine nucleotides. Sci Rep. 2015; 5: 17206.

10. Shi T, Ma Y, Cao L, Zhan S, Xu Y, Fu F, et al. B7-H3 promotes aerobic glycolysis and chemoresistance in colorectal cancer cells by regulating HK2. Cell Death Dis. 2019; 10: 308.

11. Wu J, Wang F, Liu X, Zhang T, Liu F, Ge X, et al. Correlation of IDH1 and B7H3 expression with prognosis of CRC patients. Eur J Surg Oncol. 2018; 44: $1254-1260$

12. Yao Z, Zou C, Zhou H, Wang J, Lu L, Li Y, et al. Delta(1)-pyrroline-5carboxylate/glutamate biogenesis is required for fungal virulence and sporulation. PLoS One. 2013; 8: e73483.

13. Meng Z, Lou Z, Liu Z, Hui D, Bartlam M, Rao Z. Purification, characterization, and crystallization of human pyrroline-5-carboxylate reductase. Protein Expr Purif. 2006; 49: 83-87.

14. Boer1991.pdf.

15. Gao Y, Luo L, Xie Y, Zhao Y, Yao J, Liu X. PYCR1 knockdown inhibits the proliferation, migration, and invasion by affecting JAK/STAT signaling pathway in lung adenocarcinoma. Mol Carcinog. 2020; 59: 503-511.

16. Wang QL, Liu L. PYCR1 is Associated with Papillary Renal Cell Carcinoma Progression. Open Med (Wars). 2019; 14: 586-592. 